\title{
Aplicação de Realidade Aumentada Móvel para Apoio ao Ensino de Crianças
}

\author{
Reidner Santos Cavalcante ${ }^{1}$, Flávia Gonçalves Fernandes ${ }^{1}$, \\ Edgard Afonso Lamounier Júnior ${ }^{1}$, Alexandre Cardoso ${ }^{1}$ \\ ${ }^{1}$ Faculdade de Engenharia Elétrica - Universidade Federal de Uberlândia (UFU) \\ Uberlândia, MG - Brasil \\ reidnerscloutlook.com, flavia.fernandes92@gmail.com, \\ lamounier@ufu.br, alexandre@ufu.br
}

\begin{abstract}
Initially, it is known that education is crucial for human development. Therefore, researchers are increasingly seeking more advanced technological innovations in order to improve the quality of teaching and learning of the population. Thus, the application of Augmented Reality (AR) in education has been evaluated intensively in recent years and been highlighted, as it represents new challenges and opportunities, with the inclusion of additional information and / or relevant to the actual scenario. This work presents a proposal of application whose goal is to develop a system to support the literacy of children. This work presents techniques and applications using virtual and augmented reality in education. In this way, we hope to contribute in motivating students through technological innovations, creating a pleasant and harmonious learning environment, and effectiveness in children's learning.
\end{abstract}

Resumo. Inicialmente, sabe-se que a educação é crucial para o desenvolvimento humano. Por isso, pesquisadores buscam inovações tecnológicas cada vez mais avançadas a fim de melhorar a qualidade de ensino e aprendizagem da população. Desse modo, a aplicação da Realidade Aumentada (RA) na área da educação tem sido avaliada de forma intensiva nos últimos anos e merecido destaque, pois representa novos desafios e potencialidades, com a inserção de informações complementares elou relevantes ao cenário real. Assim, este trabalho apresenta uma proposta de aplicação cujo objetivo é o desenvolvimento de um sistema para apoio no ensino de crianças. Assim, este trabalho apresenta técnicas e aplicações que utilizam realidade virtual e aumentada na área da educação. Desta forma, espera-se contribuir na motivação de alunos, através das inovações tecnológicas, gerando um ambiente educacional agradável e harmonioso, além da eficácia no aprendizado infantil.

\section{Introdução}

Assim como várias áreas estão em constante mudança para se adaptar as novas tecnologias, a área da educação também sofre constantes mudanças na forma de ensino e o uso de tecnologias como a Realidade Virtual (RV) e a Realidade Aumentada (RA) são fontes de várias dessas mudanças.

No mundo onde a tecnologia já faz parte do cotidiano de todos, utilizar a Realidade Aumentada no ensino de crianças permite que elas possam ter um aprendizado mais 
dinâmico e possam aprender com animações ao invés de imagens em um livro. A Realidade Aumentada (RA) é uma tecnologia nova que pode ser utilizada para aplicações em diversas áreas e está em constante crescimento, sendo uma subárea da realidade virtual. Também é uma tecnologia dependente de processamento em tempo real e, por isso, é influenciada pela evolução da computação, tanto do ponto de vista do hardware quanto do software [Cardoso, Lamounier e Tori, 2001].

O desenvolvimento de aplicações de Realidade Aumentada utilizando dispositivos móveis proporciona um ambiente virtual tridimensional mais atrativo e interessante para facilitar as tarefas do dia-a-dia, auxiliar diagnósticos e tratamentos de doenças, além de facilitar o aprendizado e comunicação de crianças e adultos [Lamounier e Cardoso, 2004].

A partir desse conceito, dentre várias opções na área tecnológica, pode-se citar que a Realidade Aumentada (RA) tem sido bastante utilizada para a construção do conhecimento, tornando prática e dinâmica a interatividade do usuário juntamente com a participação da mobilidade oferecida pelos dispositivos móveis, tais como, smartphones e tablets [Lecheta, 2010].

É possível adquirir dispositivos de baixo custo capazes de executar aplicações que utilizam de Realidade Aumentada e usá-los dentro da sala de aula como uma ferramenta de auxílio no ensino. O uso isolado dessas tecnologias não garante resultados condizentes com o esperado, por isso elas devem usadas como um apoio e não como única forma de ensino.

Visto que o uso da Realidade Aumentada traz mais interatividade e dinamismo para as atividades desenvolvidas dentro de sala de aula, este trabalho propõe o desenvolvimento de uma ferramenta de RA para dispositivos móveis para auxiliar no ensino de crianças utilizando de modelos tridimensionais de animais, seus principais movimentos e sons que produz.

\section{Realidade Aumentada Móvel na Educação}

Alguns aparelhos de telefone celular são dispositivos que apresentam um bom poder de processamento, levando-se em conta que se tratam de dispositivos móveis microprocessados com características limitadas. Estes são capazes de executar algoritmos desenvolvidos nas linguagens $\mathrm{C}++$ e Java.

A versão Java Micro Edition (ME) define um ambiente flexível para a construção de aplicativos destinados a dispositivos móveis, como celulares, PDAs, TV set-top boxes e impressoras. O Java ME inclui interface com o usuário, segurança robusta e protocolos de comunicação em rede [Lecheta, 2010]. Um conjunto mínimo de características do Java ME deve ser suportado pelo dispositivo para este estar apto a rodar uma aplicação de RA, como: Mobile Information Device Profile 2.0, Mobile Media API, Connected Limited Device Configuration 1.1.

Um exemplo de uma aplicação voltada para a área da educação é o MagicBook (Billinghurst, Kato e Poupyrev, 2001) que permite ao usuário transitar entre o mundo real e o mundo virtual ao ler um livro de histórias, proporcionando novas experiências para os usuários no que diz respeito a forma de ler. A Figura 1 traz o livro no mundo real com marcadores seguida pela visualização utilizando a realidade aumentada e a realidade virtual. 

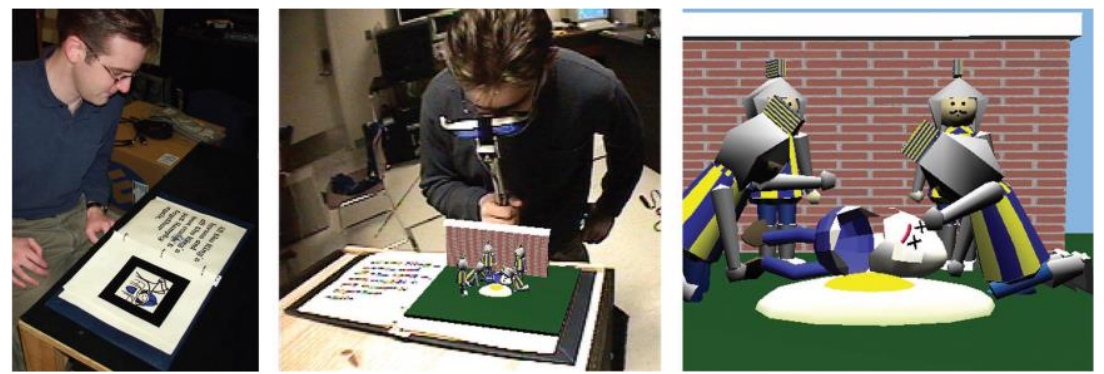

Figura 1: Visão do mundo real, realidade aumentada e realidade virtual com o MagicBook [ Billinghurst, Kato e Poupyrev, 2001].

Outro exemplo de uso da RA é o sistema de museu virtual [Kirner e Zorzal, 2005] onde marcadores são posicionados nas paredes do museu, próximo às obras. $\mathrm{O}$ aparelho celular, ou outro dispositivo móvel, ao capturar a imagem do marcador, está apto a mostrar na tela do aparelho, ou vocalmente, informações adicionais sobre a obra exposta.

Os exemplos citados falam de aplicativos que precisam estar conectados a uma rede externa através de tecnologia Bluetooth ou outro tipo de rede sem fio para obter dados adicionais. Nem todas as soluções precisam estar conectadas a uma rede externa. Um exemplo é um jogo de futebol para celular, onde uma bola virtual na tela do aparelho pode ser chutada com o próprio pé do usuário simplesmente apontando a câmera para o chão. A posição do pé é detectada em relação à bola virtual [Kirner e Zorzal, 2005].

\section{Trabalhos Relacionados}

Nesta seção serão descritos alguns trabalhos relacionados ao ambiente virtual e sua contribuição na área da educação.

O sistema AR Chemical Connection apresenta em sua interface um painel lateral que mostra a descrição das ligações químicas, facilitando a visualização das informações expostas. A Realidade Virtual e a Realidade Aumentada podem contribuir e melhorar representações simulando fenômenos como se de fato existissem naquele exato momento, proporcionando a visualização e interação do estudante com o conhecimento de modelos químicos de maneira completa, facilitando a compreensão dos conceitos químicos, ou para correção de concepções inadequadas de modelos científicos. Nesta aplicação, o usuário então coloca dois marcadores na área de captura de imagem da câmera. Em seguida o sistema, através do FlartoolKit, faz a detecção e o rastreamento dos marcadores e projeta os objetos tridimensionais sobre eles, conforme pode ser observado na Figura 2 [Araújo, 2009].

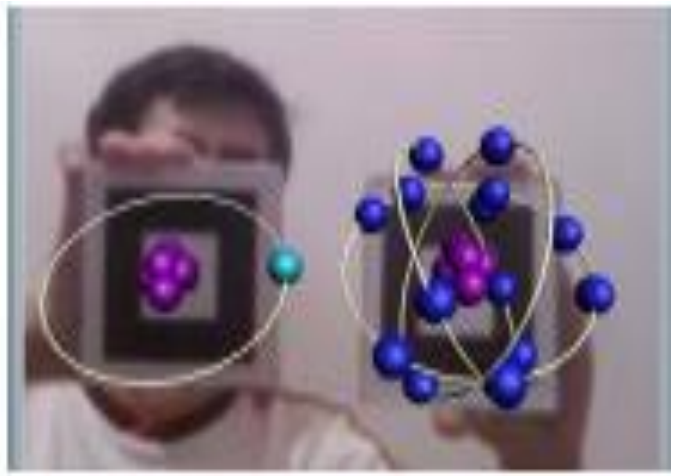

Figura 2: Objetos virtuais projetados sobre os marcadores [Araújo, 2009]. 
V Congresso Brasileiro de Informática na Educação (CBIE 2016)

Anais dos Workshops do V Congresso Brasileiro de Informática na Educação (CBIE 2016)

Um exemplo de aplicação existente que utiliza interação natural é a Fusion4D do projeto VIDA [Woodill, 2010], ilustrado na Figura 3, é um trabalho que foi desenvolvido uma aplicação 3D na qual o usuário interage com os objetos virtuais como se estivessem realmente em suas mãos, podendo mover, girar, aumentar, explodir em detalhes e até mesmo ver como os objetos seriam no passado e no futuro.

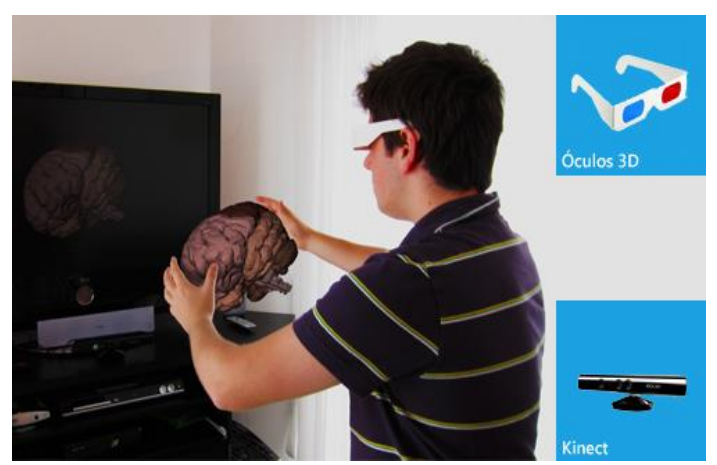

Figura 3: Projeto Fusion4D [Woodill, 2010].

Além disso, há um Sistema Interativo com Realidade Aumentada para Ensino da Anatomia (SIRAEA), onde é representada, como na Figura 4, a estrutura de um crânio tridimensional (3D) e permite aos usuários desmontar, montar e manipular o crânio 3D de forma intuitiva utilizando apenas webcam e marcador [Tori et al, 2006].

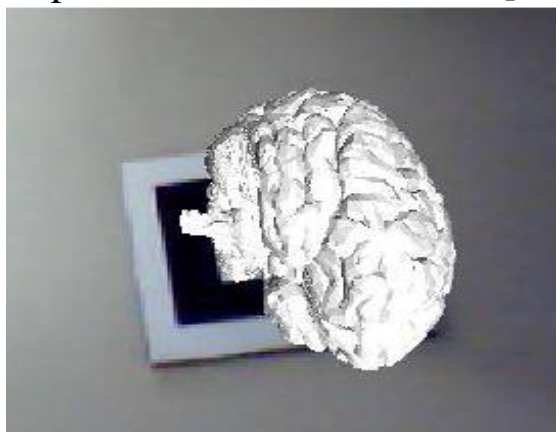

Figura 4: Cérebro virtual em Realidade Aumentada [Tori et al, 2006].

Também já foi implementada uma Aplicação Educacional aplicada à Medicina utilizando Realidade Aumentada (AEMRA), em que o seu desenvolvimento é realizado com uso da biblioteca ARToolKit. Nesse trabalho, há exemplos de sobreposição de órgãos sobre os marcadores, como o coração virtual em RA da Figura 5, que permite visualização e manipulação do respectivo órgão com aspectos diferenciados tornando o aprendizado mais dinâmico e realista [Costa e Ribeiro, 2009].

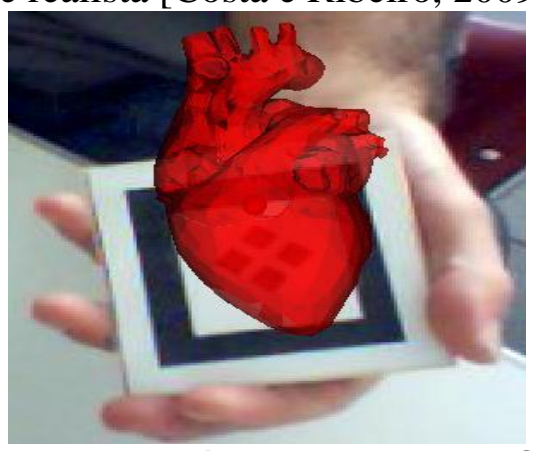

Figura 5: Coração virtual em Realidade Aumentada [Costa e Ribeiro, 2009]. 
V Congresso Brasileiro de Informática na Educação (CBIE 2016)

Anais dos Workshops do V Congresso Brasileiro de Informática na Educação (CBIE 2016)

E, ainda, foi desenvolvido um Ambiente de Realidade Aumentada para ensinoaprendizagem de Diabetes Mellitus (ARADM), que foi realizado por meio de representações tridimensionais, interativas e em tempo real, de seus principais aspectos anatômicos e fisiopatológicos, como pode ser visto na Figura 6 [Drab e Artner, 2005].

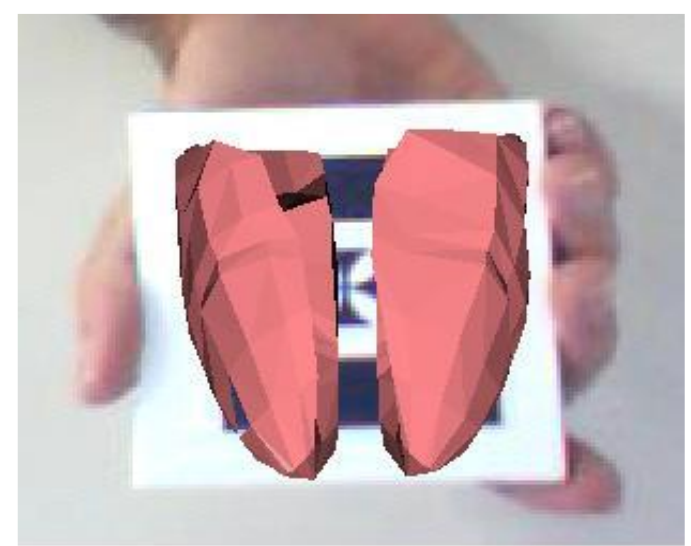

Figura 6: Rins virtuais em Realidade Aumentada [Drab e Artner, 2005].

Outra aplicação também voltada para o ensino, o Aritmética (Figura 7), pertence ao projeto SICARA [Kirner] que visa auxiliar no ensino das operações aritméticas utilizando da tecnologia de Realidade Aumentada com marcadores.
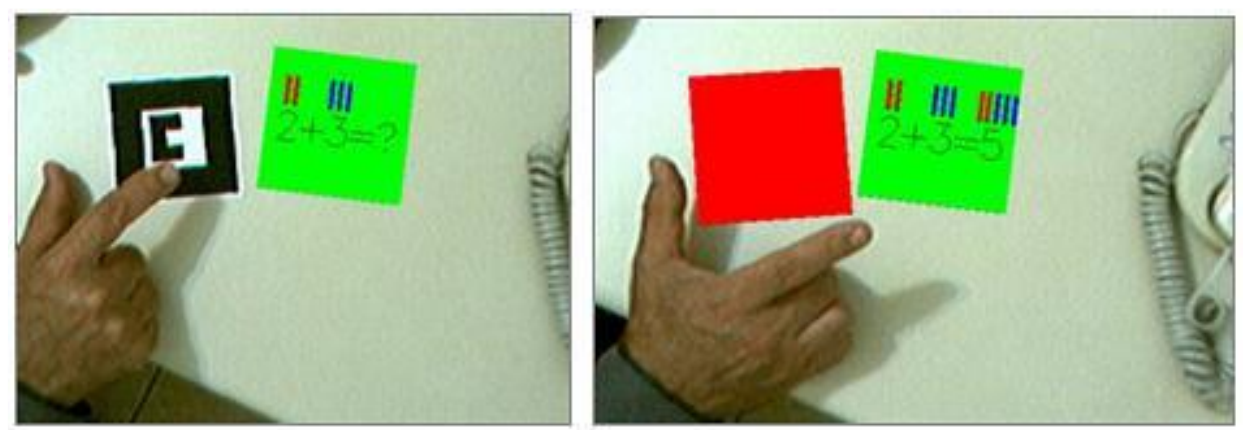

Figura 7: Projeto SICARA. Sistema de Aprendizagem de Aritmética [Kirner].

O jogo Quebra-Cabeça Ordenador [Zorzal, 2008] apresenta um jogo onde devese organizar os marcadores, que ao serem apresentados ao sistema de RA exibem números inteiros, de forma que fique organizados sequencialmente e pode ser utilizada em aulas de matemática. A Figura 8 exibe o jogo desenvolvido.
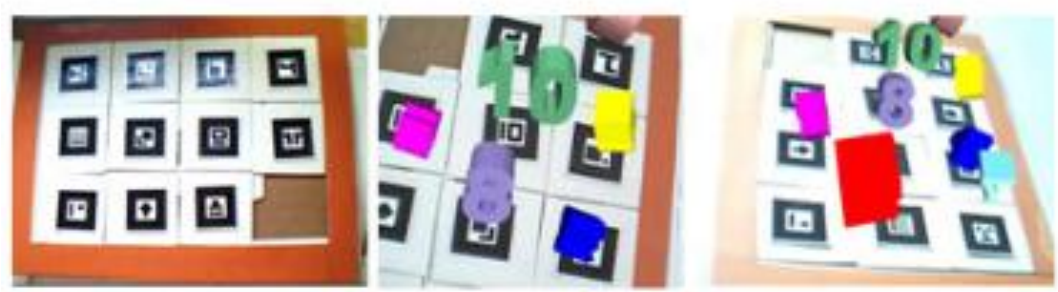

Figura 8: Cenário do quebra-cabeça ordenador [Zorzal, 2008]. 
V Congresso Brasileiro de Informática na Educação (CBIE 2016)

Anais dos Workshops do V Congresso Brasileiro de Informática na Educação (CBIE 2016)

O projeto ARAVET (Augmented Reality Application on the Field of Vocational Education and Training) [Kraut e Jeknić, 2015] apresenta três aplicações de Realidade Aumentada para as áreas de ensino e formação professional. A primeira aplicação desenvolvida é voltada para o ensino na formação de profissionais para a área de indústria têxtil, onde uma máquina de costura virtual (Figura 9) foi desenvolvida e é possível interagir com ela desde trocar sua bobina até inserir a linha na agulha.

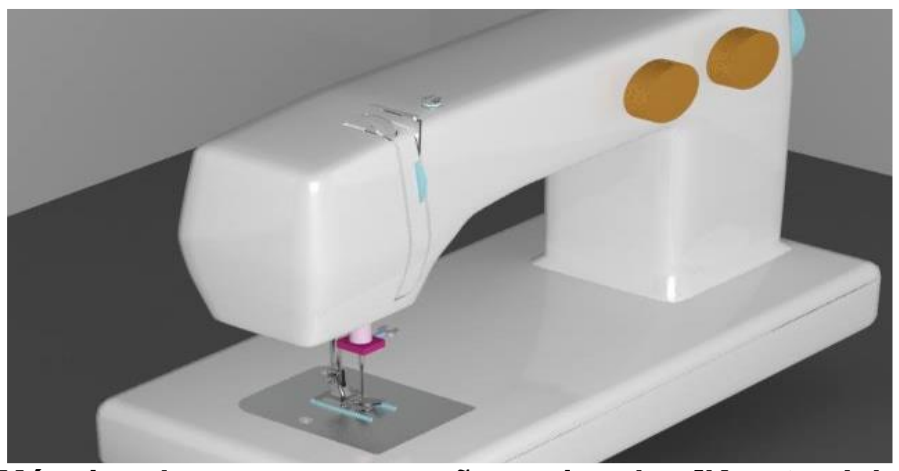

Figura 9: Máquina de costura com ações animadas [Kraut e Jeknić, 2015].

A Figura 10 apresenta outra aplicação também desenvolvida no projeto ARAVET, ela é utilizada no ensino de eletrônica exibindo o ciclo de operação de um diodo. É possível interagir com a aplicação de forma a reverter a corrente elétrica e ver as alterações que o funcionamento do diodo sofre, permitindo um aprendizado mais dinâmico e interativo dentro da sala de aula.

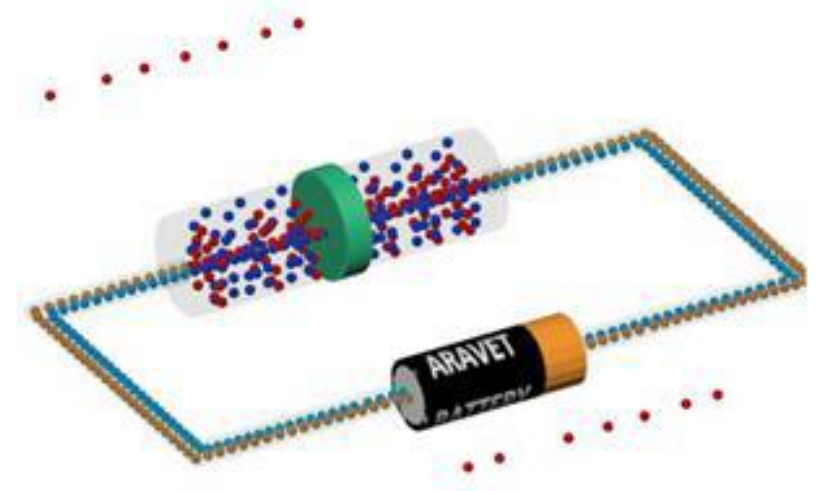

Figura 10: Comportamento do diodo e fluxo de elétrons [Kraut e Jeknić, 2015].

Outra aplicaçação também voltada para o ensino é aprensentada na Figura 11. A aplicação é voltada par ao ensino de conceitos lógicos e portas lógicas (AND, OR, XOR e NOR) utilizando a tecnologia de Realidade Aumentada. 


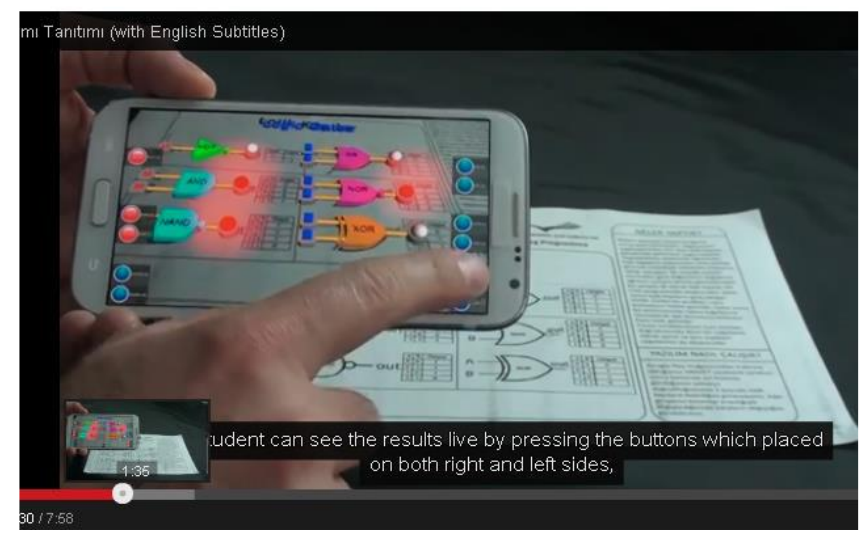

Figura 11: Elementos virtuais lógicos [Kraut e Jeknić, 2015].

\section{Aplicação de RA Móvel Aplicada no Ensino de Crianças}

Este trabalho propõe o desenvolvimento de uma aplicação para auxílio no ensino de crianças utilizando RA para dispositivos móveis.

Esse sistema tem como objetivo auxiliar crianças que estão nos primeiros anos da escola e que deve ser utilizado como uma ferramenta de apoio para fixação do conteúdo lecionado por meio da Realidade Aumentada Movél.

Utilizando de modelos tridimensionais projetos no mundo real o aprendizado das crianças se torna mais dinâmico e interativo, proporcionando um nível mais alto de aprendizado para as crianças.

Nesta aplicação, a criança poderá ter acesso em seu próprio dispositivo móvel adequado para a aplicação da RA, que exibirá as ilustrações como se fossem reais.

Em relação aos aspectos metodológicos e tecnológicos da implementação do sistema, foi utilizada RA por meio do desenvolvimento de ambientes virtuais, incluindo interações e animações, com uso da linguagem de programação Java,a aplicação será desenvolvida no ambiente Eclipse, conectado ao emulador do sistema Android e para a modelagem gráfica optou-se pela ferramenta Blender, a fim de contemplar cálculos e comandos, visando criar para o usuário a possibilidade de interagir com ambientes virtuais atrativos, que facilitem a aprendizagem significativa de conceitos teóricos e práticos. Também utiliza o sistema Android para dispositivos móveis na realização de testes e para sua utilização prática e efetiva [Harma, 2003].

O emulador Android, também conhecido como Android Virtual Device (AVD), realiza o reconhecimento de características de smartphones usando a versão do Android ${ }^{\mathrm{TM}}$ escolhida (seja a 2.1, 2.2 ou qualquer outra). Com ele, são carregados a imagem do sistema e características, onde o usuário pode realizar testes no projeto [Lecheta, 2010].

O Android SDK é um aplicativo instalado no software Eclipse que permite que os desenvolvedores elaborem as aplicações a partir de um dispositivo virtual para os aparelhos de celular e tablet, desde jogos a utilitários que façam uso das funções oferecidas pelos aparelhos, como touchscreen, telefonia GSM, Câmera, GPS, bússola, acelerômetro, Bluetooth, EDGE, 3G e WiFi. A plataforma apresenta suporte para mídias de áudio, vídeo e imagem, nos formatos MPEG4, H.264, MP3, AAC, AMR, JPG, PNG, GIF, bem como acelerador gráfico 3D, baseados no OpenGL ES. Os dados podem ser 
V Congresso Brasileiro de Informática na Educação (CBIE 2016)

Anais dos Workshops do V Congresso Brasileiro de Informática na Educação (CBIE 2016)

armazenados em SQLite e a plataforma traz um navegador integrado com base no código livre do motor WebKit [Neto, 2006].

Além disso, foi utilizada a biblioteca ARToolKit para a implementação desta aplicação. Ela é open source e viabiliza o desenvolvimento de interfaces para realidade aumentada. Esta ferramenta emprega métodos de visão computacional para detectar tags na imagem capturada por uma câmera. O rastreamento óptico desta tag possibilita o ajuste de posição e orientação para realizar a renderização de um objeto virtual, de modo que esse objeto pareça estar junto a tag, desta forma o usuário pode manipular o objeto virtual, utilizando um objeto real [Azuma, 2011].

Ao instalar o aplicativo em um celular que tenha sistema operacional Android, o usuário deve escolher um animal dentre os disponíveis e apontar a câmera do dispositivo para o marcador, após realizar essas etapas o item selecionado é exibido em Realidade Aumentada. A Figura 12 ilustra a projeção de um animal selecionado para ser exibido em RA.

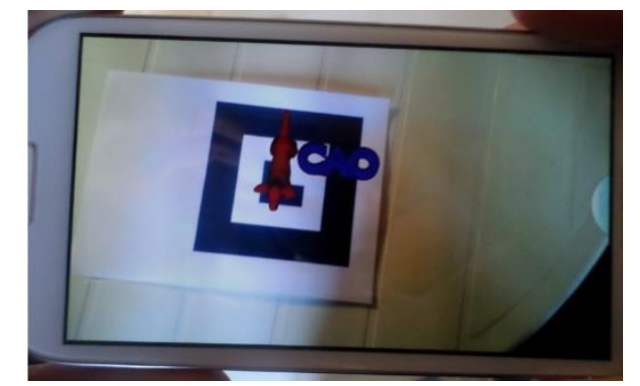

Figura 12: Cão exibido em RA no celular.

Tanto o objeto virtual quanto o seu nome são exibidos em Realidade Aumentada, deixando mais fácil a assimilação do objeto ao seu nome o que proporciona um melhor nível de aprendizado. As Figuras 13(a) e 13(b) exibem outros animais em Realidade aumentada.

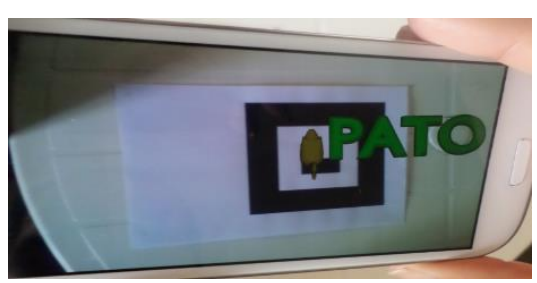

(a)

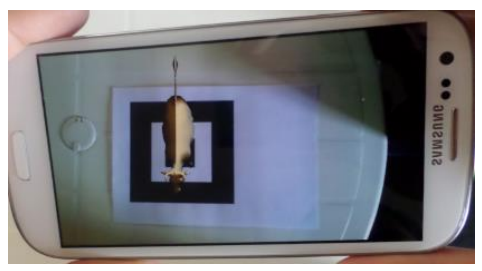

(b)

Figura 13: (a) Pato exibido em RA.

(b) Vaca exibida em RA.

A plataforma Android atualmente é tecnologia predominante nos dispositivos móveis e de baixo custo que vem ganhando grande espaço no mercado tecnológico. Também compõem este aplicativo as imagens tridimensionais criadas e desenvolvidas na plataforma Blender que dispõe de uma variedade ferramentas para manipular e criar imagens em 3D com contornos e perfeitos [Blender, 2015].

Para o desenvolvimento da ferramenta foi utilizado o conceito da visão pelo vídeo ou tela do dispositivo móvel através da captura da imagem a partir da câmera do aparelho apontada no marcador. O marcador é predefinido no sistema que reconhece as imagens 
V Congresso Brasileiro de Informática na Educação (CBIE 2016)

Anais dos Workshops do V Congresso Brasileiro de Informática na Educação (CBIE 2016)

retornando o resultado a partir da escolha realizada pelo usuário no menu inicial do aplicativo.

Após a conclusão de desenvolvimento do aplicativo, pode-se analisar as necessidades de melhoramento para trabalhos futuros, além disso, pode-se destacar já a importância da utilidade do aplicativo no desenvolvimento da criança nos primeiros anos na escola e a aproximação da criança às novas tecnologias.

Diante dos modelos desenvolvidos, a ferramenta possui imagens do cotidiano, a assimilação da imagem e a palavra é a combinação perfeita para o entendimento do usuário.

O sistema consegue reconhecer o marcador a partir de qualquer superfície plana ou não com ambiente de luminosidade clara ou natural.

\section{Conclusões}

Com base nas informações apresentadas nota-se que a Realidade Aumentada é uma tecnologia excepcional e que pode ser aplicada a diversas áreas, principalmente na educação.

A RA é uma área tipicamente multidisciplinar que envolve conceitos provenientes de diversos segmentos, com aplicação em várias áreas ou campos específicos da vida econômica, social e cultural. Por permitir uma interação humano-computador mais natural em ambiente tridimensional (3D) e possibilitar a reprodução de situações reais, torna-se um recurso de amplo potencial.

O uso da RA em dispositivos móveis proporciona aos usuários a possibilidade de ter uma aplicação que pode ser executada a qualquer momento e garante uma grande mobilidade, sendo preciso apenas um dispositivo com android e câmera para funcionar. .

A partir de suas características de usabilidade, dinamismo, capacidade de promover a interação entre os mundos real e virtual, torna a capacidade de comunicar diante da disposição de se ter o conhecimento no cotidiano em qualquer lugar.

Com isso, este trabalho apresentou a utilização da tecnologia de Realidade Aumentada combinada com a aprendizagem móvel para criação de ambientes de auxílio ao ensino de crianças, permitindo ampliar as possibilidades de aprendizado de crianças.

Como proposta de melhorias futuras, propõe-se adicionar mais animais e implementar animações de forma que o usuário consiga visualizar o animal e como ele realizações como comer, dormir, andar e correr.

\section{Referências}

Araújo, D. M. et al. Uso de realidade aumentada como ferramenta complementar ao ensino das principais ligações entre átomos. In: VI Workshop de Realidade Virtual e Aumentada, Universidade de Santa Cecília, 2009.

Azuma, R.; Billinghurst, M. and Klinker, G.. 2011. "Editorial: Special Section on Mobile Augmented Reality", in Computer Graphics. pp. vii-viii.

Billinghurst, Mark; KATO, Hirkazu; POUPYREV,Ivan. The MagicBook - Moving Seamlessly Between Reality and Virtuality. IEEE Computer Graphics and Applications, vol. 21, 2001. 
V Congresso Brasileiro de Informática na Educação (CBIE 2016)

Anais dos Workshops do V Congresso Brasileiro de Informática na Educação (CBIE 2016)

Blender, 2015, [Online; accessed 20-Set-2012]. [Online]. Available: http://www.blender.org/.

Campanário, I. S. Espelho, espelho meu: A psicanálise e o tratamento precoce do autismo e outras psicopatologias graves. Salvador: Ágalma, 2008.

Cardoso, A. ; Lamounier Jr. and Tori, R. (2001), “Interactive 3D Physics Experiments through the Internet", Proceedings of the 4th SBC Symposium on Virtual Reality, pp.280-90, Florianópolis, Santa Catarina, Brazil. http://users.hub.ofthe.net/ mtalkmit/veshtml2.html, November.

Costa, R. M.; Ribeiro, M. W. Aplicações de realidade virtual e aumentada. Porto Alegre: SBC, 2009. $146 \mathrm{p}$.

Drab, S.; Artner, N M. Motion Detection as Interaction Technique for Games \& Applications on Mobile Devices. Pervasive Mobile Interaction Devices, Munich, Alemanha, 2005.

Harma, A. et al. Techniques and applications of wearable augmented reality audio. In: Audio Engineering Society Convention Paper, Amsterdam, Holanda, 2003.

Kirner, C; Zorzal, E."Jogos Educacionais em Ambiente de Realidade Aumentada". Workshop de Realidade Aumentada, Piracícaba- São Paulo, 2005.

Kirner, Cláudio. Projeto SICARA. Disponível em: http://www.ckirner.com/claudio/?PROJETOS:SICARA. Acesso em: 20 de agosto de 2016.

Kraut, B. \& Jeknić, J. Improving education experience with augmented reality (AR). 2015 38th Int. Conv. Inf. Commun. Technol. Electron. Microelectron. MIPRO 2015 - Proc. 755-760 (2015). doi:10.1109/MIPRO.2015.7160372

Lamounier, E. e Cardoso, A. Realidade virtual: uma abordagem prática. São Paulo: Mania de Livro, 2004. 326 p.

Lecheta, R. R.. Google Android, 2nd ed., Novatec, Ed., 2010. [Online]. Available: http://www.livroandroid.com.br/

Milgram, P. et. al. (1994) "Augmented Reality: A Class of Displays on the RealityVirtuality Continuum". Telemanipulator and Telepresence Technologies, SPIE, V.2351, p. 282-292.

Neto, S. et al. Criação de Aplicações de Realidade Aumentada em Dispositivos Móveis Baseados em Symbian OS. In: II Workshop de Aplicações de Realidade Virtual, Universidade Federal de Pernambuco, 2006, p. 16-19.

Tori, R.; Kirner, C.; Siscouto, R.."Fundamentos e Tecnologia de Realidade Virtual e Aumentada”. Belém: VIII Symposium On Virtual Reality, 2006.

Woodill, G. "The Mobile Learning Edge: Tools and Technologies for Developing Your Teams", 1st ed., McGraw-Hill, Ed., 2010. [Online]. Available: http://www.mobilelearningedge.com.

Zorzal, E. R. et al. Aplicação de Jogos Educacionais com Realidade Aumentada. RENOTE, v. 6, n. 2, 2008. 